\title{
Eco-Driving Assistance System for Manual Transmission Bus Based on Machine Learning
}

\author{
Hongjie Ma, Hui Xie, David Brown, Member, IEEE
}

\begin{abstract}
Driving Assistance Systems (DAS) is a key technology to improve fuel-economy for in-use vehicles. This also reduces the operational cost of running a fleet of these vehicles, such as city buses. In this paper, we develop a novel white box evaluation model using machine learning for manual transmission bus based on previous research about fuel consumption sensitivity to driving style. Using the proposed evaluation model, an algorithm for Learning Path Planning (LPP) for a driving style is also proposed. The LPP method plans a step-by-step shortest learning path for different driving styles to achieve eco-driving while increasing the driver's acceptance and adaptation of DAS. Simulation results based on vehicle and engine physical models show that the proposed evaluation model, a pure data model, can be used as an alternative to physical model for the eco-driving prompt strategy. The results of the verification show that the proposed strategy can progressively guide the driver to improve the fuel consumption by $6.25 \%$ with minimal changes to driver's driving task and driving style.
\end{abstract}

Index Terms - Eco-driving, driving style, driver evaluation, driving assistance system, decision tree.

\section{INTRODUCTION}

Eco-driving is an economical and ecological style of driving. Adopting this style of driving can lead to better fuel economy and lower emissions of vehicles. Research has shown that eco-driving has the potential to save about $25 \%$ fuel and this saving is approximately three times the fuel saving that can be made through engine tuning and tire optimization [1]. Survey results have also shown that drivers are keen to benefit from this style of driving [2]. However, there was minimal effort in past from manufacturers and from research community to explore and promote the eco-driving[3]. Considering the significance of eco-driving for fuel economy, this technology has developed rapidly in recent years and current research on eco-driving can be classified into optimization at three levels: a) planning and optimization at task level, b) optimization at strategy level and c) optimization at operations level[4].

Hongjie Ma Tianjin University and Institute of Industrial Research, University of Portsmouth (e-mail: hongjie.ma@port.ac.uk).

Hui Xie (Corresponding author) is now with Tianjin University, 92 Weijin Rd, Nankai Qu, China. (e-mail: xiehui@tju.edu.cn).

David Brown is now with department of Institute of Industrial Research, University of Portsmouth, Unit 1, St Andrew's Court, PO12PR, United Kingdom. (e-mail: david.j.brown@port.ac.uk).
The research on the planning and optimization at task level mainly focuses on route planning for fuel economy. The driving routes that $46 \%$ of the vehicles choose do not achieve the best fuel economy because the route with the shortest time or shortest distance may not necessarily be the best one for fuel economy $[5,6]$. The fuel consumption for the shortest time route could be $9.3 \%$ more than the best fuel economical route [7]. A route optimization method using ergodic optimization approach is presented and the approach can achieve up to $8.2 \%$ savings in fuel consumption for a 5 miles driving route [5]. Similar approach considering different type of vehicle is presented in [8]. However, these approaches are for the vehicles on non-fixed routes, but not for vehicles on fixed routes such as buses or logistics vehicles.

Planning and optimization at strategy level focuses on optimizing the control strategy of Engine Control Unit (ECU) to match the ECU's performance with road conditions and/or driving style for better fuel economy. An automatic optimization of engine calibration parameters for different driving styles based on a self-learning controller is presented in [9] and this method optimizes the matching of engine's energy consumption characteristics with driving styles by adjusting the parameters such as injection timing and cycle injection quantity, etc. Modeling of engine's operational conditions using Markov Decision Process to optimize the engine's control parameters of for different driving styles is presented in [10]. Although these methods are promising for eco-driving, but they are only applicable for depth customization and optimization of the vehicle control system at the design stage currently.

Optimization at operation level focuses on optimizing the driver's operational with the aid of Driver Assistant Technology (DAS) to improve driving style. This optimization approach to eco-driving has a wider application and greater potential as compared to the other two optimization approaches for in-use vehicles. The reason is that average consumption varies for different driving styles by up to $30 \%$ in the urban environment and $17 \%$ for highway [11]. Driving styles also have a significant influence even on the energy consumption of electric vehicles. Bingham et al. found that the difference in the energy consumption between mild and radical driving styles is about $30 \%$ [12].

The optimization at operations level can be achieved either by training the drivers of vehicles or by using real-time DAS 
technology[13, 14]. Both these approaches have good effect on fuel economy [15]. Driver training was widely adopted by public transportation and logistics companies before the advent of real-time DAS technology and such training can lead to savings in fuel consumption up to $11.6 \%$ [16]. Some of these training rules derive from the experience of well-behaved drivers, while others are derived from statistical analysis of big data technologies[17].

Real-time DAS technology gives real-time assistance to the driver through graphical units or sound to improve fuel economy. This technology can also be combined with traditional training method to further improve the fuel economy[14]. Real-time DAS typically consists of prompt algorithm and a Human Machine Interface (HMI). The prompt algorithm can be divided into two categories: speed planning algorithm and operational planning algorithm. The speed planning algorithm outputs the target speed in real-time for driver to follow while the operational planning algorithm provides the guidance on real-time operation. An optimal speed algorithm using dynamic planning based on the vehicle model and engine fuel economy map is presented in [18]. In addition to the vehicle configuration, traffic information is also important for the speed planning and [19] used the traffic information as a constrained boundary for planning the optimal speed profile. Similar approach for planning the speed profile during deceleration process based on the traffic light information is presented in [20]. The premise of speed planning algorithms is to assume that the eco-speed profile corresponds to eco-operations. This assumption is very harsh because the same speed profile can be achieved by the different combination of operations. This will lead to different fuel consumption for different drivers, although they follow the same speed profile.

The algorithms based on the operational planning require more parameters and they are difficult to obtain using physical models. The reason for this is that in an engine fuel consumption model, it is very hard to achieve balance between complexity and precision[21]. Since each engine type has its own unique model, therefore this also limits the application of physical model based operational planning algorithms. At present, most operational planning algorithms provide operational feedback with reference to driver's 'normal operation' by making statistics on the driver's historical on-road driving data [22]. However, the 'normal operation' is not necessarily the best operation at all times, and such algorithm may lead to a driving style with worse effect on fuel economy. Some of the more intelligent algorithms are based on the fuzzy logic or Bayesian algorithm and historical on-road driving data for each user to provide personalized assistance[23, 24].

Another challenge facing DAS technology is the wider acceptance of this technology. Higher the difference between an DAS's guidance information relative to the inherent driving habits and style of the driver leads to a lowered the acceptance/adoption of the DAS guidance by the driver. To address this issue, a closed-loop speed advisory model to generate the refuel economy speed profile is presented[25]. However, for the DAS technology with algorithms for operational planning, an approach that takes the driver's learning ability and adoption into account has not been developed. In this paper, we address this challenge and develop operational planning algorithm while taking into consideration the driver's leaning and DAS adoption into account.

The selection of HMI for DAS is also a focus of research. The HMI of DAS typically includes visual, auditory, visual-auditory[26] and haptic[27]. The deployment costs, driver performance, the acceptance and workload associated with the interaction are the four criteria for evaluating different HMIs[28]. The workload of the haptic-based solution is better than the visual-auditory based solution, while the other three criteria are the opposite[29]. Despite this, the workload of the visual-auditory based solution is much smaller than navigation or the operation of the change $\mathrm{CD}[30]$. Therefore, we choose the visual-auditory as the HMI in this research.

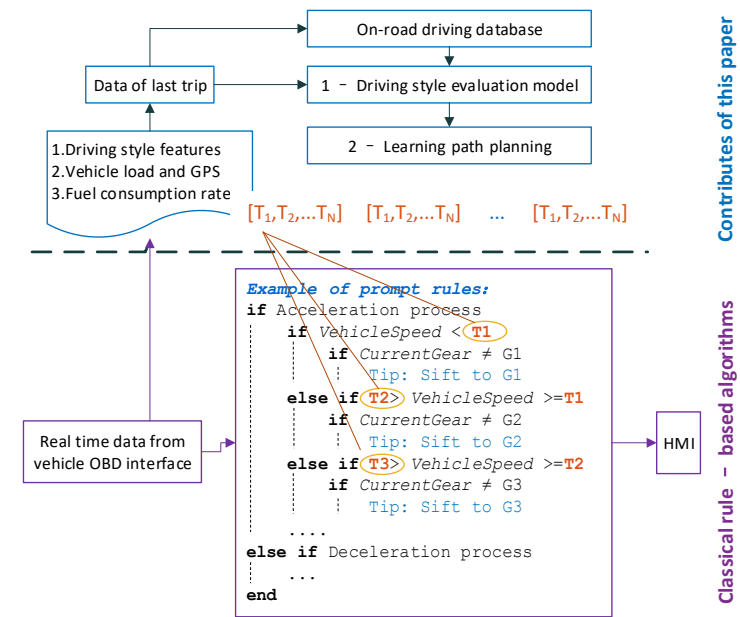

Fig. 1. Structure of Eco-Driving Assistance System with driving style evaluation model and learning path planning.

As shown in Fig. 1, the driver's operations and the corresponding fuel consumption rate are collected via the OBD interface. Based on these raw data, the vehicle load (including slope and vehicle mass), driving style characteristic parameters (as shown in the Table 1) and the corresponding fuel consumption rate are calculated. These three types of interrelated data form an individual historical sample. Although the random factors such as congested traffic, traffic lights, road conditions, wind speed and wind direction etc. have an impact on fuel consumption for each individual sample, it is necessary to note that under the premise of sufficient samples the impact of these random factors on fuel consumption will be excluded. Therefore, a machine learning is adopted to build the driving style evaluation model based on the large number of historical samples (section II. A). After that a learning path planning (LPP) algorithm based on the evaluation model is proposed to provide the thresholds for the classic rule-based 
Eco-driving assistance algorithm, which could help the driver to achieve better fuel economy with the real-time prompts (section II. B). Finally, the precision of the driving style evaluation model and the effectiveness of the LPP algorithm are verified based on a co-simulation platform (section III).

\section{AlgORITHM FOR ECO-DrIVING ASSISTANCE}

TABLE 1

DRIVING STYLE CHARACTERISTIC PARAMETERS OF MANUAL TRANSMISSION BUS.

\begin{tabular}{|c|c|}
\hline Abbreviation & Remark \\
\hline G1toG2Speed & Shift speed at gear $1 \rightarrow 2$ of driven process \\
\hline G2toG3Speed & Shift speed at gear $2 \rightarrow 3$ of the driven process \\
\hline G3toG4Speed & Shift speed at gear $3 \rightarrow 4$ of the driven process \\
\hline $\begin{array}{l}\text { AvgAcPedG1, AvgAcPedG2, } \\
\text { AvgAcPedG3, AvgAcPedG4 }\end{array}$ & $\begin{array}{l}\text { Average depth of accelerator pedal in gear } 1 \text {, } \\
2,3 \text { and } 4 \text { of driven process }\end{array}$ \\
\hline $\begin{array}{l}\text { AvgAcPedDiff }+ \text { G1, AvgAcPedDiff }+\mathrm{G} 2 \text {, } \\
\text { AvgAcPedDiff }+\mathrm{G} 3 \text {, AvgAcPedDiff }+\mathrm{G} 4\end{array}$ & $\begin{array}{l}\text { Average positive gradient of accelerator } \\
\text { pedal in gear } 1,2,3 \text { and } 4 \text { of driven process }\end{array}$ \\
\hline
\end{tabular}

In our previous studies, as shown in Table 1 a set of characteristic parameters describing driving styles related to fuel economy for manual transmission bus driver were studied and the influence of these parameters on the fuel consumption under different traffic conditions and loads was analyzed. This parameter set consists of shift timings, average accelerator pedal depth, and average accelerator pedal's positive rate of change for each gear. It was also established that the influence of the driving style on the fuel consumption of manual transmission bus under specific circumstances could be obtained based on at least $2500 \mathrm{~km}$ on-road driving data [31]. These characteristic parameters are the basis for building the driving style evaluation model. Based on these feature parameters, we have carried out the following innovative work:

a) A driving style evaluation model is developed based on machine learning algorithm and on-road driving data;

b) A LPP algorithm that could gradually guide the driver to improve driving behavior is developed based on the evaluation model.

The model can determine the fuel economy level of a driver for a given vehicle configuration according to the characteristic parameters of the driving style, and the LPP can plan a learning path for the driver to achieve the fuel economy upgrade with as little operational changes as possible. According to the path, the driver can gradually improve the operating habits and increase the acceptance and adoption degree of the driving DAS with an improved fuel economy.

\section{A. Driving style evaluation model of manual transmission bus drivers}

Driving style evaluation model is a classification model with multi-coupling inputs. The model takes the driving style characteristic parameters as the inputs and generates the corresponding fuel economy levels as the outputs. In this paper, the machine learning is adopted to construct the model, and the machine learning algorithm needs to have the following characteristics to meet the requirements of LPP:
1. The evaluation process is traceable, which means that the model should be a white-box model. The model should provide the classification result and the determination process of the fuel economy at the same time. There are three typical decision trees (ID3, C4.5, C5.0) could meet this requirement.

2. The algorithm should support default attributes to support the conditions where only parts of inputs are available. Such as the drivers cannot express all the operating characteristics in some traffic conditions. ID3 can't meet this requirement.

3. The calculation/complexity need of the algorithm should be as low as possible. The model may run in an embedded system with relatively weak computing power.

Both C4.5 and C5.0 decision trees could meet the requirements. The improvement of $\mathrm{C} 5.0$ compare to $\mathrm{C} 4.5$ is the modelling speed. The advantage of the $\mathrm{C} 4.5$ algorithm is that it is an open source algorithm compared to C5.0. In our study, we adopted C4.5 for driving style evaluation model[32].

$$
\begin{gathered}
\mathrm{H}(D)=-\sum_{i=1}^{n} p_{i} \log _{2}^{p_{i}} \\
g_{R}(D, A)=\frac{H(D)-H(D \mid A)}{H(D)} \\
\mathrm{H}(D \mid A)=\sum_{i=1}^{k} p_{i} H\left(D \mid A=T_{i}\right)
\end{gathered}
$$

The modeling data come from the aforementioned historical samples. The C4.5 algorithm is described in equation 1-3, where $g_{R}(D, A)$ is the increase rate of the system information entropy, if the driving style characteristic parameter $A$ is split. $D$ represents the historical sample data set consisting of driving style and corresponding fuel consumption, $p_{i}$ in equation 1 represents the probability that the fuel economy level belongs to level $L_{x}$ in all samples, $n$ represents the total number of fuel economy levels divided by users, $k$ represents the number of possible values for $A, p_{i}$ in equation 3 represents the probability that $A=T_{i}, T$ and $H$

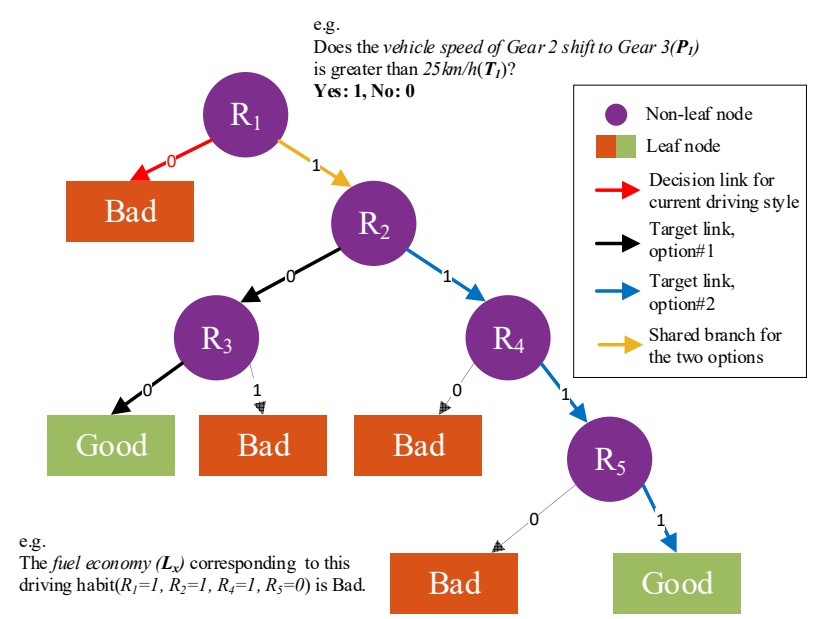

Fig. 2. An example of a driving style evaluation model based on decision 


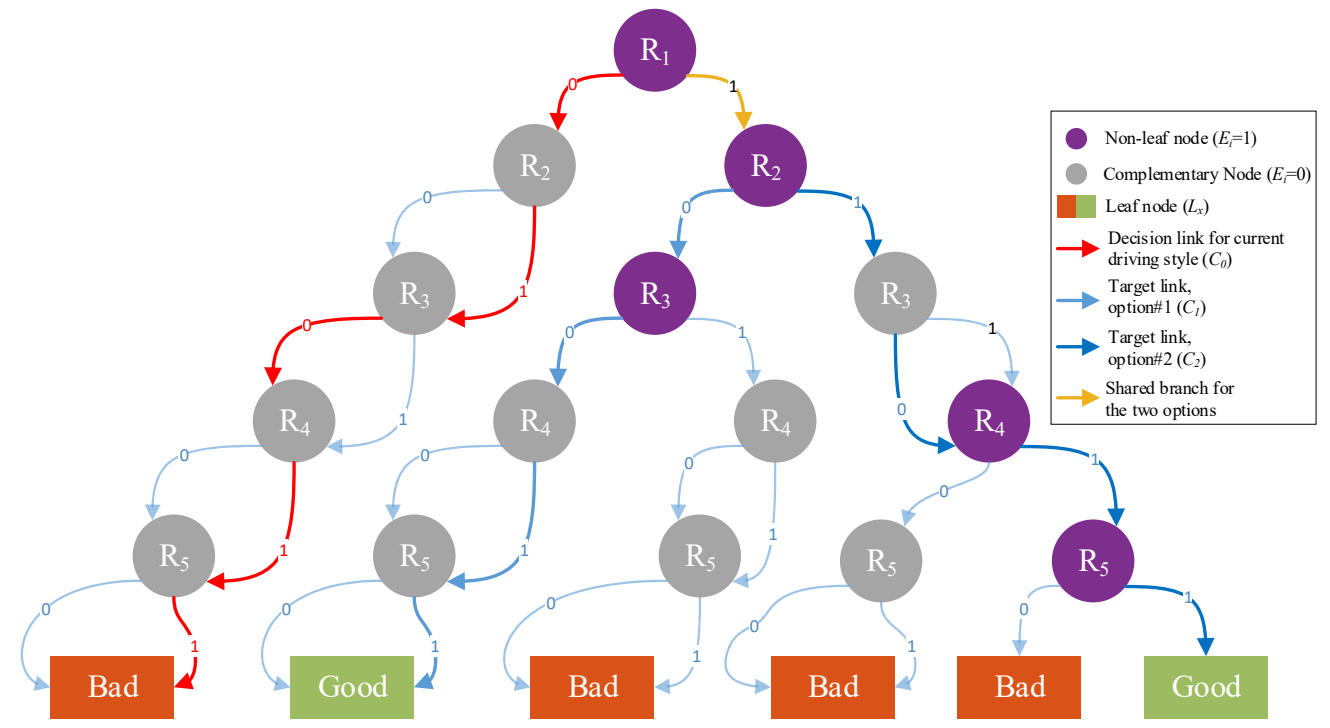

Fig. 3. Filled decision tree model of the example shown in Fig. 2.

represent the discriminative threshold of $A$ and the uncertainty of the decision system, respectively [33].

The schematic diagram of the model structure constructed by the decision tree $\mathrm{C} 4.5$ is shown in Fig. 2, where $R_{x i}$ is a non-leaf node, as shown in equation 4 , representing the binary discriminative result of the driving style characteristic parameters $\left(P_{x i}\right)$. For example, "Whether the G2toG3Speed is greater than $25 \mathrm{~km} / \mathrm{h}$ " is a non-leaf node $\left(R_{1}\right)$, in which, the G2toG3Speed is the characteristic parameter $\left(P_{1}\right)$ of the driving style, and $25 \mathrm{~km} / \mathrm{h}$ is the discriminative threshold $\left(T_{1}\right)$ of this node. The discriminant result of the node determines the branches of the tree. After a series of determination of the non-leaf nodes, the decision tree can give a leaf node $L_{x}$ (Good or Bad in Fig. 2) that represents the fuel economy level corresponding to the driving style. From this example, the traceable white-box feature of the decision tree could be found. Such as the fuel economy corresponding to the current driving style and the potential target driving style with better fuel economy can both be reflected in the model. This means that the driver from the decision link $\left[\mathrm{R}_{1}=0, \mathrm{Bad}\right]$, adjusted to target link option $1\left[\mathrm{R}_{1}=1, \mathrm{R}_{2}=0, \mathrm{R}_{3}=0\right.$, Good] or option $2\left[\mathrm{R}_{1}=1, \mathrm{R}_{2}=1, \mathrm{R}_{4}=1, \mathrm{R}_{5}=1\right.$, Good $]$ can achieve better fuel economy. This is the basis for the LPP algorithm.

\section{B. Learning path planning}

It can be found from Fig. 2 that the driver has two options to achieve better fuel economy (Good). So, the LPP algorithm should have the ability to select the better option which is the closest one to the current driving style and could gradually prompt the selection to the driver. This will reduce the changes in the driver styles and enhance the acceptance of Eco-driving assistance system.

\section{1) Selection of the target decision link}

To select the decision link that is closest to the current driving style from the decision-link set with best fuel economy, the distance between the decision links need to be measured. The method proposed in this paper is to fill the decision tree to make all decision branches with the same length. This allows the distance between the different decision links to be measured.

The Fig. 3 is the filled decision tree model of the example shown in Fig. 2. In the figure, the red color decision link stand for the driving style before using the Eco-Driving assistance system. In this decision link the non-leaf nodes $R_{2}$, $R_{3}, R_{4}$ and $R_{5}$ are not involved in the selection of the target decision link. As shown in the Fig. 3, in the filled decision model, the current decision link is $\left[\mathrm{R}_{1}=0, R_{2}=1, R_{3}=0, R_{4}=1\right.$, $\left.R_{5}=1, \mathrm{Bad}\right]$ and the two optional target links are $\left[\mathrm{R}_{1}=1, \mathrm{R}_{2}=0\right.$, $\mathrm{R}_{3}=0, R_{4}=1, R_{5}=1$, Good $]$ and $\left[\mathrm{R}_{1}=1, \mathrm{R}_{2}=1, R_{3}=0, \mathrm{R}_{4}=1\right.$, $\mathrm{R}_{5}=1$, Good]. Although the second option in the example of Fig. 2 has a longer link than the first option, it's easy to find that the driver only needs to change an operation $\left(\mathrm{R}_{1}=0 \rightarrow\right.$ $\left.\mathrm{R}_{1}=1\right)$ for the second one, while two operations $\left(\mathrm{R}_{1}=0 \rightarrow\right.$ $R_{1}=1$ and $R_{2}=1 \rightarrow R_{2}=0$ ) for the first one. So, the second option is actually better than the first option.

$$
\begin{aligned}
& R_{x i}=\left\{\begin{array}{ll}
1 & P_{j} \leq T_{x i} \\
0 & P_{j}>T_{x i}
\end{array}, i \in[1, m], j \in[1, t], x \in[1, k]\right. \\
& E_{x i}=\left\{\begin{array}{cc}
1 & \text { Effective } \\
0 & \text { Not effective }
\end{array}, i \in[1, m], x \in[1, k]\right. \\
& R_{x}=\left[R_{x 1}, R_{x 2}, \ldots, R_{x m}\right], x \in[1, k] \\
& E_{x}=\left[\begin{array}{cccc}
E_{x 1} & 0 & 0 & 0 \\
0 & E_{x 2} & 0 & 0 \\
0 & 0 & \ldots & 0 \\
0 & 0 & 0 & E_{x m}
\end{array}\right], x \in[1, k] \\
& C_{x}=\left[R_{x} E_{x}, L_{x}\right], x \in[1, k], L_{x} \in\left\{L_{1}, L_{2}, \ldots, L_{n}\right\} \\
& D T_{\text {model }}=\left\{C_{1}, C_{2}, \ldots, C_{k}\right\}
\end{aligned}
$$


TABLE 2

SYMBOL DESCRIPTION OF THE DECISION TREE MODEL.

\begin{tabular}{cl}
\hline \hline Symbol & \multicolumn{1}{c}{ Remark } \\
\hline$t$ & Number of inputs (driving style parameters) to the model \\
$m$ & Number of non-leaf nodes to the tree model \\
$k$ & Number of leaf nodes to the model \\
$n$ & Number of outputs (fuel economy levels) \\
$R_{x i}$ & Discriminant result of the non-leaf nodes \\
$C_{x}$ & Decision-making link consisting of all non-leaf nodes and one leaf node \\
$E_{x i}$ & Whether the $R_{x i}$ is involved to determine the leaf node of $C_{x}$ \\
$L_{x}$ & Leaf node, which stands for fuel economy level, corresponding to $C_{x}$ \\
\hline \hline
\end{tabular}

The mathematic representation of the aforementioned example is as equation 4-8. The symbols' descriptions are shown in Table 2 . The decision tree can be converted into the set of decision links $D T_{\text {model }}$ as shown in equation 9.

$$
\begin{gathered}
\mathrm{TG}=D T_{\text {model }} \mid \max \left(L_{x}\right) \\
D S_{x \rightarrow y}=\sqrt{\sum_{i=1}^{k}\left(R_{x i}-R_{y i}\right)^{2} E_{y i}} \\
\mathrm{TL}=\mathrm{TG} \mid \min \left(D S_{x \rightarrow y}\right), \mathrm{y} \in \mathrm{TG}
\end{gathered}
$$

The criteria to determine the goal are twofold: the fuel economy achieves the optimal value in the historical data; and the changes in the driving style are as little as possible. As shown in the equation 10, firstly, several paths with the optimal fuel economy grade are selected from the decision link set to form the target set TG. It is clear that the final learning goal of the driver learning must be included in the set TG. Then, based on the second criterion, the target TL hat is nearest to the current driving style in the target set TG is solved.

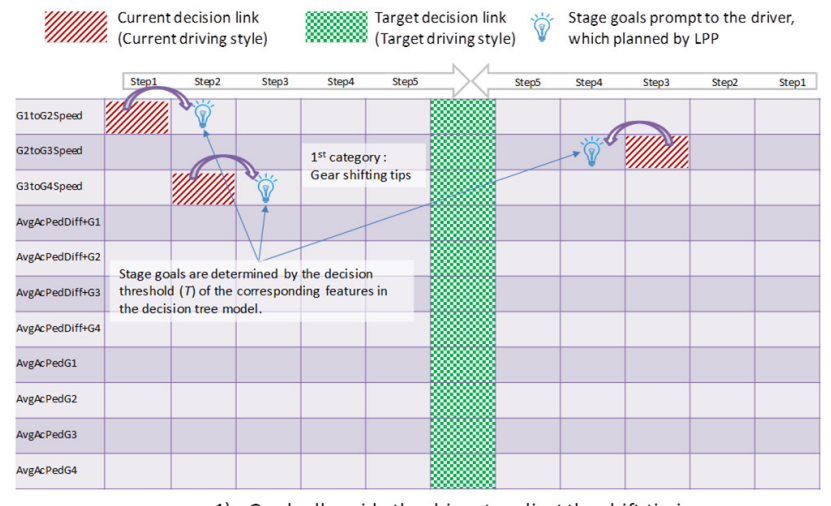

1) - Gradually guide the driver to adjust the shift timing

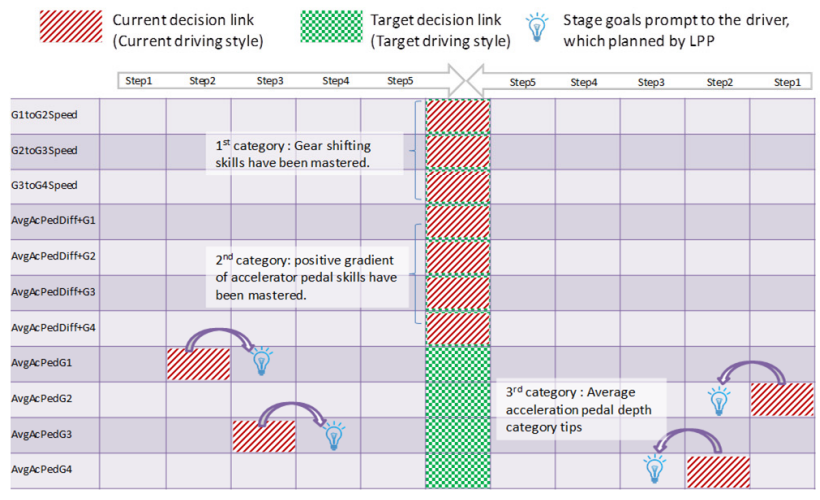

3) - Gradually guide the driver to adjust the average accelerator pedal depth
In order to measure the difference between driving styles, as shown in equation 11, we define the distance index $D S_{x \rightarrow y}$ that characterizes the difference from the decision link $C_{x}$ to $C_{y}$. The larger the value is, the more changes in operations the driver needs to make to improve from the current link $x$ to the link $y$. This is a directional distance indicator, namely, $D S_{x \rightarrow y}$ is not equivalent to $D S_{y \rightarrow x}$. The distance index is improved based on the Euclidean distance, which introduces the distance element validity parameter $E_{y i}$ based on the Euclidean distance. Therefore, the LPP algorithm learning target TL can be expressed as equation 12 .

\section{2) Prompt of the LPP}

As the example shown in Fig. 4, LPP divides the difference between the decision link which stands for the current driving style and the target decision link into three categories. They will be prompted to the driver in turn. The rules are as follows:

a) The three categories are gear shift prompts, positive gradient of accelerator pedal prompts and average acceleration pedal depth prompts, respectively. The priority of the three categories of prompts is that gear shift $>$ positive gradient of accelerator pedal $>$ average acceleration pedal depth.

b) Only one category of prompts would be given to the driver at a time to reduce the distraction of driver. This means that the LPP does not provide other categories of prompts to the driver before the previous prompt is completed.

c) Each feature in a category has the same priority and will

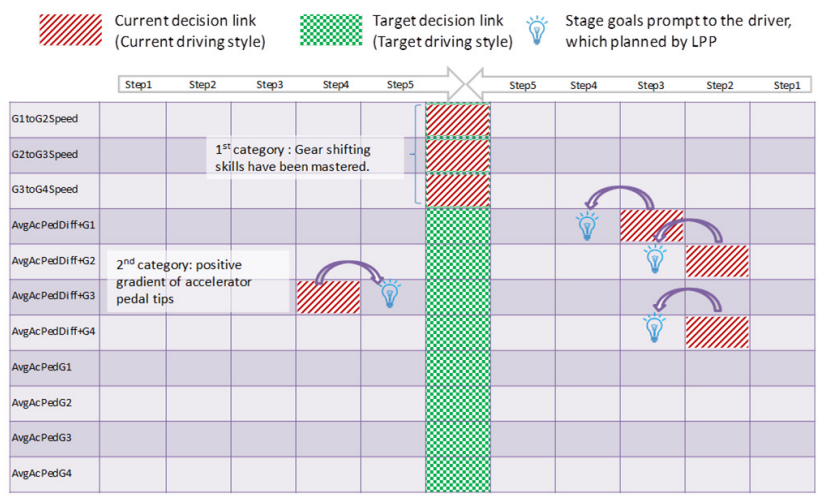

2)- Gradually guide the driver to adjust the positive gradient of accelerator pedal

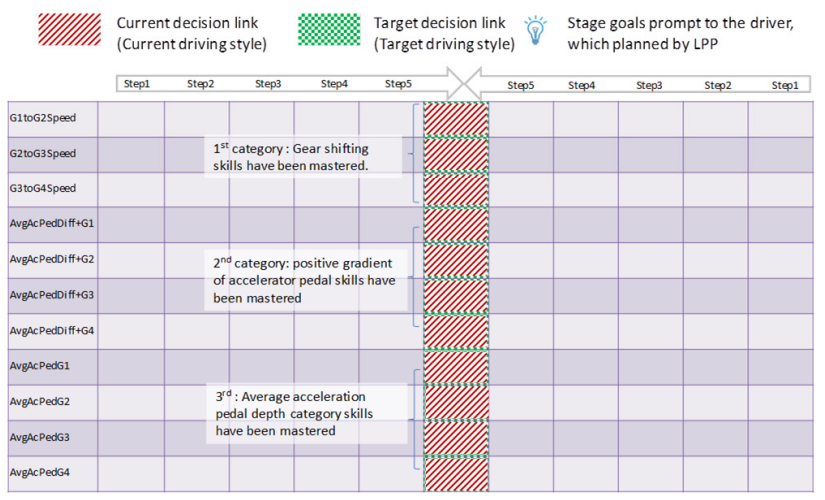

4) - Re-implement LPP based on new historical data 
be presented to the driver at different stages of a driving process. Take the gear shift prompts as an example, the three gear shift timings are prompted to the driver at three different speed points in a drive process.

d) The stage goal of each feature in a certain category is determined by the decision threshold of the corresponding feature in the decision tree model.

The implementation of the promotion is consisted by following steps:

a) The on-board unit load a file, which contains all the tips, during the start-up period. This file is updated by the LPP algorithm which running on the remote server. The updated cycle is about 20 days, which is determined by the mileage of the vehicle running.

b) The tips that have been mastered by the driver would be skipped for a short time after they start driving.

c) The tips, which have been mastered before but been forgotten at this moment, will be prompted again in the next loop.

\section{Implementation of the system}

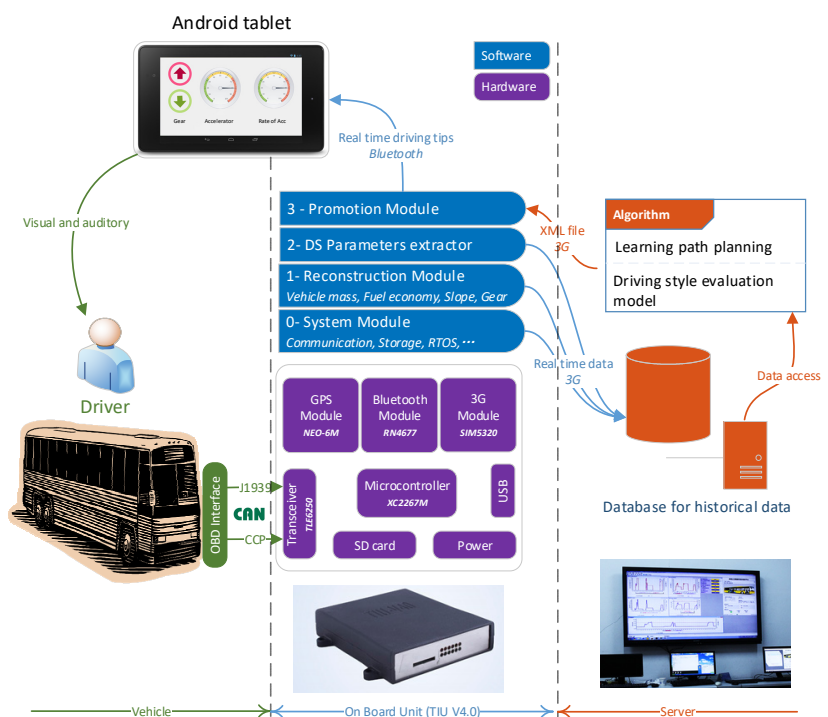

Fig. 5. An example of a driving style evaluation model based on decision tree algorithm.

The implementation of the Eco-Driving assistance system is shown in Fig. 5. The system consists of three parts: Android tablet, On-board unit and the remote server. The key part of this system is the on- board unit. As shown in the figure, the workflow of the system is as follows:

[1] The on-board unit communicates with the electronic control units of both vehicle and engine over J1939 and CAN calibration protocols.

[2] These real-time data are used to reconstruct vehicle mass, road slopes, fuel economy and gear index in the reconstruction module.

[3] After that the raw data and the reconstructed data are used to extract the driving style parameters.

[4] All the above data along with the GPS location information is transmitted over the wireless network to the remote server.

[5] Data during wireless network disconnection is cached on the local SD card and re-uploaded to the server after network recovery.

[6] The XML file generated by the algorithm running on the remote server is downloaded to the unit for promoting.

[7] Real-time driving tips are transferred to the tablet over the Bluetooth. The tips are presented to the driver in the manner of visual-auditory.

\section{RESULTS AND DISCUSSION}

In order to verify the effectiveness of the proposed algorithm, the paper has conducted the verification in two aspects: firstly, the precision of the driving style evaluation model is verified, and then the effectiveness of the LPP algorithm is verified.

\section{A. Verification for driving style evaluation model}

The data for the driving style evaluation modeling was obtained from three buses with same configurations running on the same bus route. There are 13 drivers were involved during the 19-month data collection and they were scheduled equally. The sampling interval of the data is 100 milliseconds. The data set contains 147.2 million records, covering 102,200 km of mileage. The same route and same configuration of vehicle can eliminate the influence due to the vehicle types, traffic light and traffic conditions. The $100 \mathrm{~ms}$ sampling interval can fully retain the operating characteristics of the driving style and the 13 drivers alternately driving three vehicles can rule out the influence of occasional weather or passenger load fluctuation factors on the results and analysis.

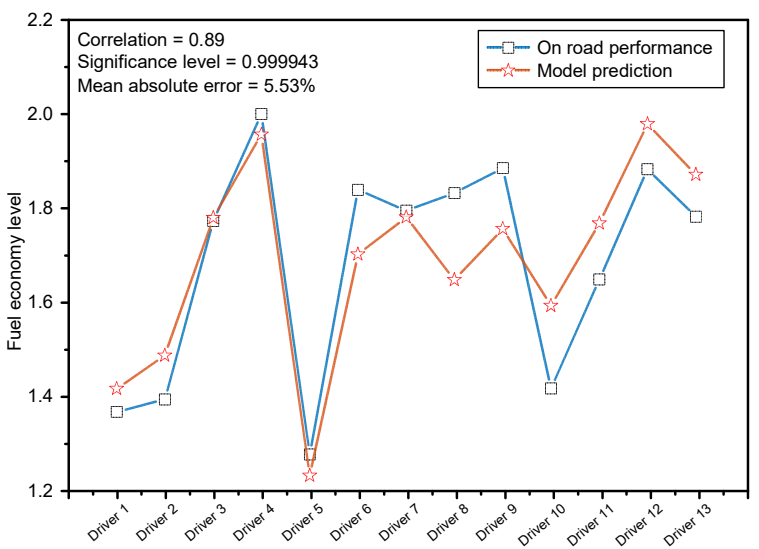

Fig. 6. The comparison between the actual fuel economy level of 13 drivers and the model predictive value.

As described above, for fixed-route manual transmission bus, $2500 \mathrm{~km}$ of operating data can reflect the impact of driving style on fuel consumption. Considering the data set contains 13 drivers, $30 \%$ of the total records $(30,660 \mathrm{~km}$ in total, $2358 \mathrm{~km} /$ driver) is adopted to build the driving style evaluation model, while the other $70 \%$ of the samples for verification purposes. In these drivers, the shortest bus driving experience is 9 years, the longest is 25 years. The 
bus company provided them with a salary incentive for Eco-Driving. Even for these motivated and driving experienced drivers, their absolute fuel consumption difference is still up to $14 \%$, which also shows that the Eco-driving assistance system has a good market prospects. By comparing the average predicted fuel economy level of each driver with the actual fuel economy level, the precision of the driver evaluation model is verified. As shown in Fig. 6, the average absolute error of the evaluation model is $5.53 \%$. In addition, it can be seen from the figure that, the predicted trend of average fuel economy level is closely matches the actual average fuel economy level with the correlation of 0.89 between the two with corresponding significance level of 0.999943 . This means that the driving style evaluation model, constructed based on the $\mathrm{C} 4.5$ decision tree algorithm, can achieve the expected objectives.

\section{B. Verification for $L P P$}

In this paper, the described LPP algorithm is tested in the co-simulation platform constructed by GT-SUITE ${ }^{\circledR}$ and Simulink $^{\circledR}$, where the detailed physical models are implemented in the GT-SUITE, and the driving style evaluation model and LPP algorithm are implemented in the Simulink ${ }^{\circledR}$.

\section{1) Simulation platform}

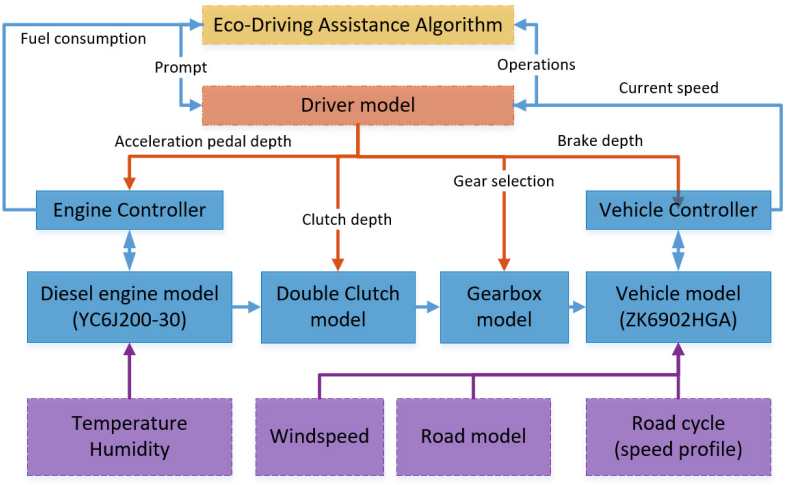

Fig. 7. Simulation platform for LPP verification.

The architecture of the simulation model adopted in the verification is shown in Fig. 7, including four sub-models: powertrain, driver, vehicle and environment. The powertrain sub-model includes the engine control, gear box and engine model. The vehicle sub-model includes the vehicle controller and vehicle longitudinal dynamic model, while the environment sub-model includes the inputs of wind speed, temperature and road, etc. The driver model supports the import of the actual driver's combined operations to simulate the corresponding fuel economy behavior of the real driver operation. The driver model also supports the collaboration with the LPP algorithm in the Simulink environments to simulate the driver's expected response to the prompt of the LPP algorithm.

The vehicle model used for energy consumption research verifies the running resistance which includes air resistance

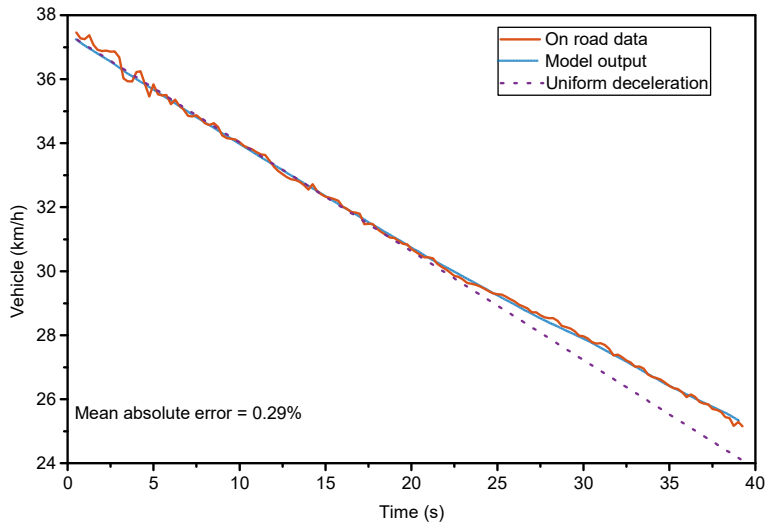

Fig. 8. Accuracy validation of vehicle model.

and rolling resistance. The rolling resistance of the vehicle is determined by the vehicle mass, and the air resistance is determined by the vehicle speed. Model calibration ensure that the deceleration speed profile of the vehicle during the free-run is consistent with the actual profile. As shown in Fig. 8, the vehicle is free to coast for 40 seconds from 37.4 $\mathrm{km} / \mathrm{h}$. The average error between the profile of the simulated model and the measured data is $0.29 \%$.

The engine and its controller model are calibrated according to the experimental data of the manufacturer, and the

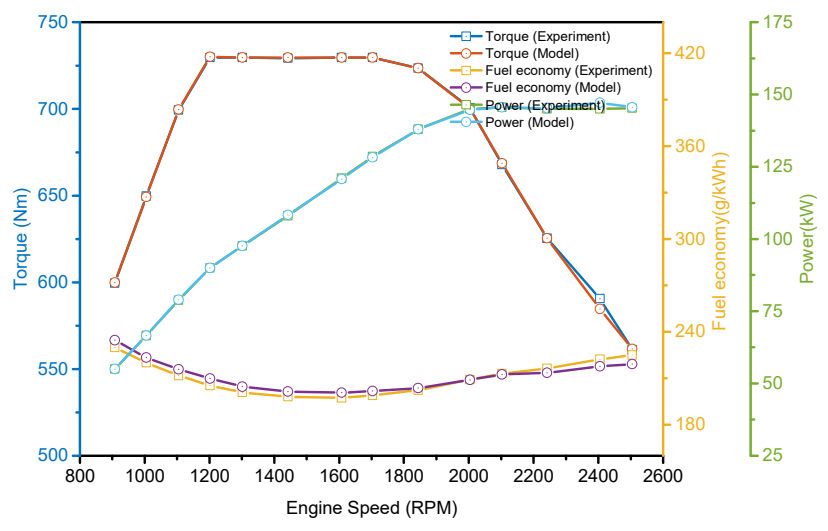

Fig. 9. Accuracy validation of engine simulation model.

calibration results are shown in Fig. 9. It can be seen that the average error of the external characteristic torque of the model is $0.12 \%$, and the average error of fuel economy is $1.51 \%$. The fuel injection characteristic map of the engine and the driving characteristic map in the controller model adopt the calibration data of a real engine electronic control unit. The dynamic response characteristic and fuel economy characteristic of the engine are consistent with the ones of the original engine.

Since the power train model and the vehicle model demonstrate good validation accuracies, therefore the aforementioned simulation platform is used to verify the LPP algorithm. 


\section{2) Verification results of $L P P$ algorithm}

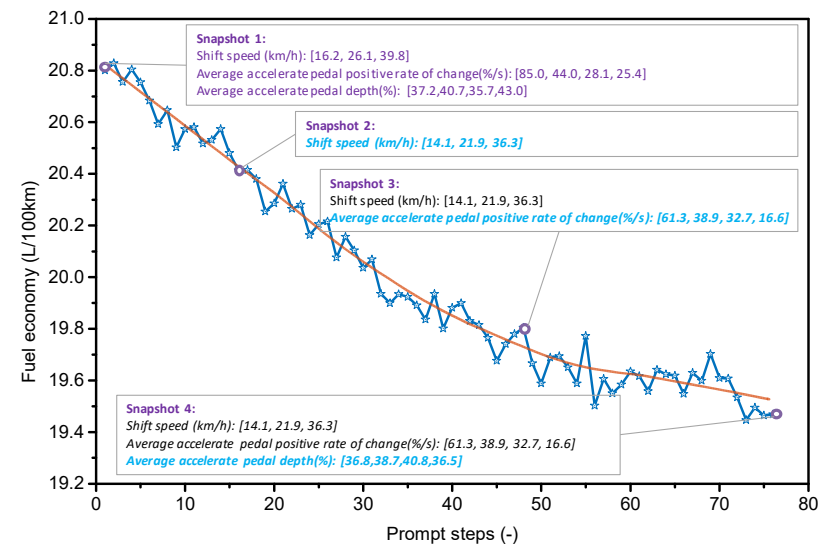

Fig. 10. Fuel economy of each step planned by LPP.

In order to verify the effectiveness of the LPP algorithm, we selected a 250 s driving segment of the actual operation as a benchmark to simulate the acceptance and adaptation of the LPP tips to gradually achieve the better fuel economy. The driving segment includes three typical processes: 1) continuous acceleration from stop to maximum speed, 2) constant speed and 3) deceleration, which is helpful for evaluating the proposed algorithm under different scenarios. As shown in Fig. 10, the number of tips which LPP prompts is 76 , which means that there are 76 fine-tuning steps between the current driving style and the ideal one. These steps can be further divided into three categories, where the shift speed class includes 16 adjustment steps, the accelerator pedal change rate class includes 32 adjustment steps, and the average accelerator pedal depth includes 28 adjustment steps. Snapshots before and after operation adjustments are used for comparison. Comparison of snapshot 1 and snapshot 2 shows the shifting speeds of 4 gears are changed from $16.2 \mathrm{~km} / \mathrm{h}, 26.1 \mathrm{~km} / \mathrm{h}, 39.8 \mathrm{~km} / \mathrm{h}$ to $14.1 \mathrm{~km} / \mathrm{h}, 21.9 \mathrm{~km} / \mathrm{h}, 36.3 \mathrm{~km} / \mathrm{h}$ respectively. This suggests that the shift timings of the initial driving style are late for all gears. Similarly, as shown in snapshot 3 and snapshot 4 , the accelerator pedal change rate class and the average

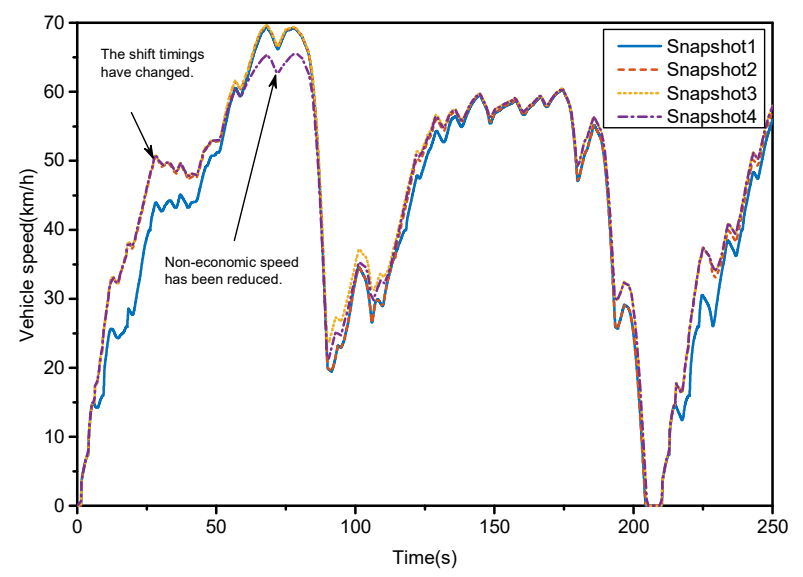

Fig. 11. Contrastive analysis of vehicle profiles corresponding to the four snapshots.

accelerator pedal depth are also adjusted. As also shown in the figure, after the above adjustment, the fuel economy of the vehicle is improved by $6.25 \%$.

As shown in Fig. 11, the vehicle speed profiles of four snapshots are basically the same, indicating that LPP has little effect on the driving task. The comparison of snapshots 1 and 2 shows that the deviation of the speed profile is greater in the acceleration process than in the other phases, as a result of the shift timing change. Snapshot 2 and snapshot 3 speed profile basically coincide, indicating that the accelerator pedal change rate adjustment strategy provided by LPP has little effect on vehicle speed. The comparison of snapshot 3 and snapshot 4 shows that the speed of snapshot 3 has been reduced in the range of $62 \mathrm{~s} \sim$ $85 \mathrm{~s}$, indicating that the speed of this period is not efficient for the engine.

In order to further analyze the principle of LPP algorithm to achieve fuel-saving, we compared the distributions of engine operating point for the four snapshots. As shown in Fig. 12, changes in driving behavior will directly lead to the engine operating point to the trajectory of significant changes, although the final speed curve is essentially the same. The different distribution of engine operating point will lead to different fuel efficiency. By comparing the boxplot of snapshot 1 and 2. it can be seen from the Fig. 12 that the median engine speed was reduced from $1458 \mathrm{rpm}$ to $1388 \mathrm{rpm}$, and the torque was increased from $346 \mathrm{Nm}$ to $376 \mathrm{Nm}$ due to a change in shift timing. This change in distribution will cause the engine operating conditions to move towards more fuel-efficient areas. The comparison of snapshot 2 and 3 shows that although the statistical distribution of torque and rotational speed is essentially the same, which shown in the boxplots, the fluctuation of the trajectory of the latter engine is significantly reduced. A reduction in engine operating point fluctuations can reduce the additional fuel consumption caused by engine transient behavior. Figure 12 also shows that the average depth of the accelerator pedal is reduced for snapshot 4 , the operating point of the low fuel efficiency zone at engine speeds of more than $1794 \mathrm{rpm}$ is significantly reduced. From this we can conclude that: Firstly, the pure data model based on machine learning method can give the guidance of eco-driving instead of the physical model, and secondly, that LPP can guide the driver to improve the fuel economy step by step.

\section{CONCLUSION AND FUTURE WORK}

Based on the C4.5 decision tree, a white-box evaluation model of driving style is developed. The results of 13 drivers showed that the predicted results were consistent with the trend of the on road driving data, showing high correlation (0.89), with good significance level (0.999943). This shows that the pure data model based on machine learning has the ability to express the fuel economy level of the driver. On the basis of this model, a LPP for improving the driving style is proposed, which takes a directional distance as indicator. With help of proposed LPP, the driver can achieve the best fuel economy with minimal change in driving style. 

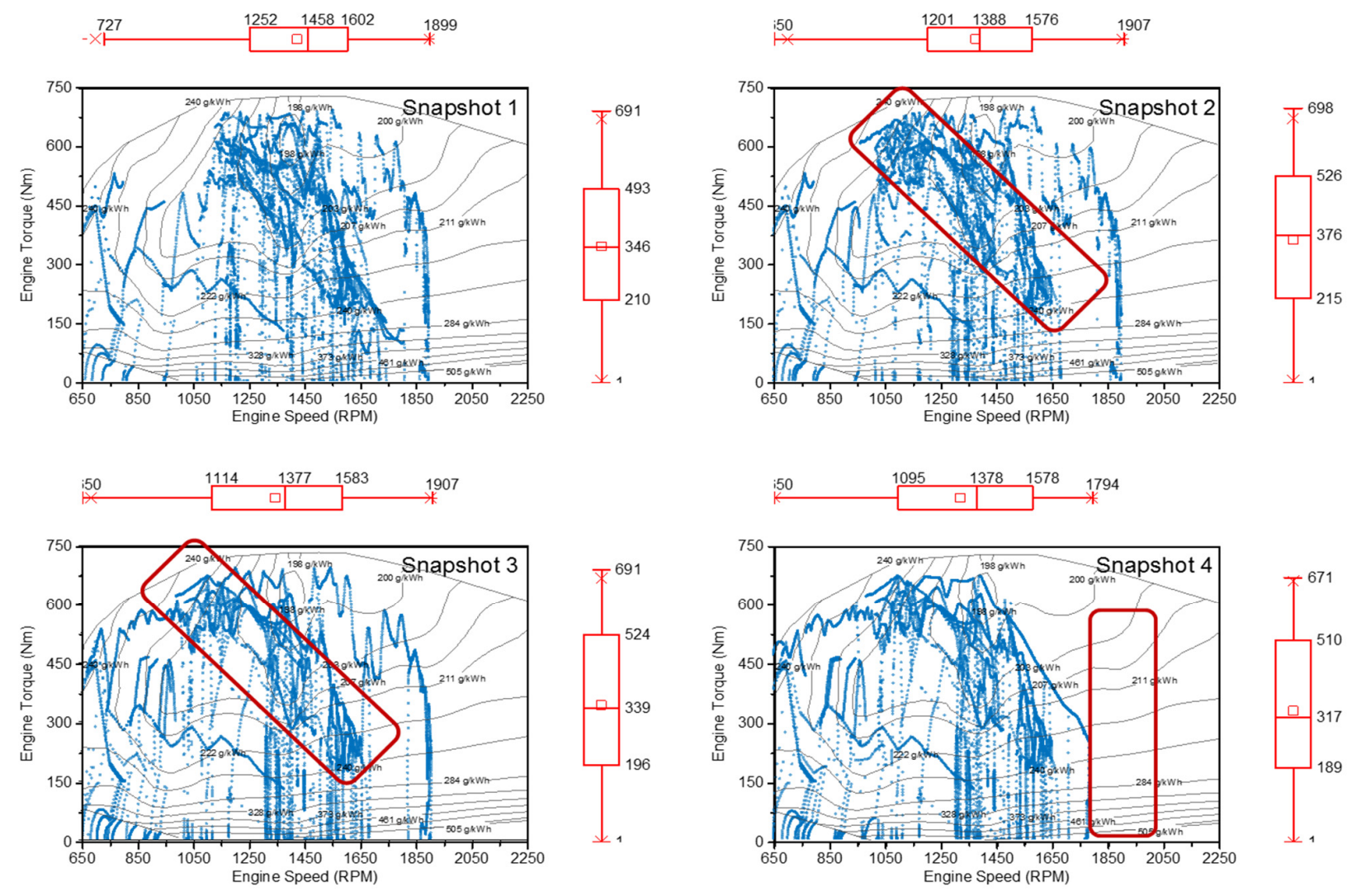

Fig. 12. Contrastive analysis of engine operating points distribution corresponding to the four snapshots.

Based on the simulation results of vehicle and engine simulation platform, the LPP algorithm can guide the driver to increase the fuel economy by $6.25 \%$ under the premise that the driving task is basically unchanged. Further analysis of the engine operating point trajectory reveals that the proposed LPP could help the driver to improve fuel economy from three aspects: a) moving the engine operating points distribution to the better fuel economy region with changed the shift timings; b) reducing the engine transient behavior with limited the accelerator pedal's positive rate of change; c) reducing the driving behavior which beyond the economic speed. The analysis result proves the validity of the driving assistant optimization algorithm.

The performance of our proposed algorithm may further be enhanced by: a) carrying out tests in further application scenarios, such as non - fixed route vehicles, to improve the universality of the algorithm, b) integration with control strategy of ECU to enhance its self-optimization capabilities, c) evaluating the fuel saving performance of the algorithm under the real scenarios.

\section{REFERENCES}

[1] Sivak M, Schoettle B. Eco-driving: Strategic, tactical, and operational decisions of the driver that influence vehicle fuel economy. Transport Policy 2012;22:96-9.

[2] Fors C, Kircher K, Ahlström C. Interface design of eco-driving support systems - Truck drivers' preferences and behavioural compliance. Transportation Research Part C: Emerging Technologies 2015;58:706-20.
[3] Barkenbus JN. Eco-driving: An overlooked climate change initiative., 2010: 762-9

[4] Lee C, Lee C, Tsai H. An economic assistance strategy for autonomous driving system. Advanced Robotics and Intelligent Systems (ARIS), 2015 International Conference on; 2015: IEEE; 2015. p. 1-4.

[5] Ericsson E, Larsson H, Brundell-Freij K. Optimizing route choice for lowest fuel consumption - Potential effects of a new driver support tool. Transportation Research Part C 2006;14(6):369-83.

[6] Caulfield B, Brazil W, Ni Fitzgerald K, Morton C. Measuring the success of reducing emissions using an on-board eco-driving feedback tool. Transportation Research Part D: Transport and Environment 2014;32:253-62.

[7] Ahn K, Rakha HA. Network-wide impacts of eco-routing strategies: A large-scale case study. Transportation Research Part D: Transport and Environment 2013 2013-12-01;25:119-30.

[8] Ganti RK, Pham N, Ahmadi H, Nangia S, Abdelzaher TF. GreenGPS: a participatory sensing fuel-efficient maps application. Proceedings of the 8 th international conference on Mobile systems, applications, and services; 2010: ACM; 2010. p. 151-64.

[9] Malikopoulos AA, Assanis DN, Papalambros PY. Optimal Engine Calibration for Individual Driving Styles: SAE Technical Paper; 2008.

[10] Malikopoulos AA, Assanis DN, Papalambros PY. Real-time self-learning optimization of diesel engine calibration. Journal of Engineering for Gas Turbines \& Power 2009;131(2):22803.

[11] Jeffrey Gonder MEAW. Analyzing Vehicle Fuel Saving Opportunities through Intelligent Driver Feedback. 2012.

[12] Bingham C, Walsh C, Carroll S. Impact of driving characteristics on electric vehicle energy consumption and range. IET Intelligent Transport Systems 2012;6(1):29-35.

[13] Birrell SA, Fowkes M, Jennings PA. Effect of Using an In-Vehicle Smart Driving Aid on Real-World Driver Performance. IEEE Transactions on Intelligent Transportation Systems 2014;15(4):1801-10.

[14] Rolim C, Baptista P, Duarte G, Farias T, Shiftan Y. Quantification of the Impacts of Eco-driving Training and Real-time Feedback on Urban Buses Driver's Behaviour. Transportation Research Procedia 2014;3:70-9.

[15] Andrieu C, Pierre GS. Comparing Effects of Eco-driving Training and Simple Advices on Driving Behavior. Procedia - Social and Behavioral 
Sciences 2012;54:211-20.

[16] Sullman MJM, Dorn L, Niemi P. Eco-driving training of professional bus drivers - Does it work? Transportation Research Part C: Emerging Technologies 2015;58:749-59.

[17] Ferreira JC, de Almeida J, Da Silva AR. The impact of driving styles on fuel consumption: a data-warehouse-and-data-mining-based discovery process. IEEE Transactions on Intelligent Transportation Systems 2015;16(5):2653-62. [18] Nouveliere L, Braci M, Menhour L, Luu HT, Mammar S. Fuel consumption optimization for a city bus. UKACC Control Conference; 2008; 2008. p. 1-6.

[19] Mensing F, Bideaux E, Trigui R, Tattegrain H. Trajectory optimization for eco-driving taking into account traffic constraints. Transportation Research Part D: Transport and Environment 2013;18:55-61.

[20] Thijssen R, Hofman T, Ham J. Ecodriving acceptance: An experimental study on anticipation behavior of truck drivers. Transportation Research Part F: Traffic Psychology and Behaviour 2014;22:249-60.

[21] Zhou M, Jin H, Wang W. A review of vehicle fuel consumption models to evaluate eco-driving and eco-routing. Transportation Research Part D: Transport and Environment 2016;49:203-18.

[22] Orfila O, Saint Pierre G, Messias M. An android based ecodriving assistance system to improve safety and efficiency of internal combustion engine passenger cars. Transportation Research Part C: Emerging Technologies 2015;58:772-82.

[23] Gilman E, Keskinarkaus A, Tamminen S, Pirttikangas S, Röning J, Riekki J. Personalised assistance for fuel-efficient driving. Transportation Research Part C: Emerging Technologies 2015;58:681-705.

[24] Suzdaleva E, Nagy I. Data-based speed-limit-respecting eco-driving system. Transportation Research Part C: Emerging Technologies 2014;44:253-64

[25] Xiang X, Zhou K, Zhang W, Qin W, Mao Q. A Closed-Loop Speed Advisory Model With Driver's Behavior Adaptability for Eco-Driving. IEEE Transactions on Intelligent Transportation Systems 2015;16(6):3313-24.

[26] Vagg C, Brace CJ, Hari D, Akehurst S, Poxon J, Ash L. Development and field trial of a driver assistance system to encourage eco-driving in light commercial vehicle fleets. IEEE Transactions on Intelligent Transportation Systems 2013;14(2):796-805.

[27] Albers A, Schwarz A, Zingel C, Schroeter J, Behrendt M, Zell A, et al. System-oriented validation aspects of a driver assistance system based on an accelerator-force-feedback-pedal. Proceedings of the FISITA 2012 World Automotive Congress; 2013: Springer; 2013. p. 221-33.

[28] Jamson AH, Hibberd DL, Merat N. Interface design considerations for an in-vehicle eco-driving assistance system. Transportation Research Part C: Emerging Technologies 2015;58:642-56.

[29] Hibberd DL, Jamson AH, Jamson SL. The design of an in-vehicle assistance system to support eco-driving. Transportation Research Part C: Emerging Technologies 2015;58:732-48.

[30] Rouzikhah H, King M, Rakotonirainy A. Examining the effects of an eco-driving message on driver distraction. Accident Analysis \& Prevention 2013;50:975-83

[31] Ma H, Xie H, Huang D, Xiong S. Effects of driving style on the fuel consumption of city buses under different road conditions and vehicle masses. Transportation Research Part D: Transport and Environment 2015;41:205-16.

[32] Wu X, Kumar V, Quinlan JR, Ghosh J, Yang Q, Motoda H, et al. Top 10 algorithms in data mining. Knowledge and information systems 2008;14(1):1-37.

[33] Quinlan JR. C4. 5: programs for machine learning: Elsevier, 2014.

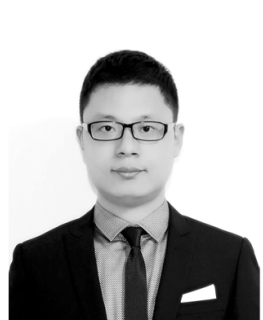

Hongjie Ma is a Senior Research Fellow in Institute of Industrial Research at University of Portsmouth. He received his Ph.D. in power machinery and engineering from Tianjin University, China, in 2015. His research focused on the electronic control and data mining based optimization for engine and vehicle. His current research interests focus on data mining and artificial intelligence.

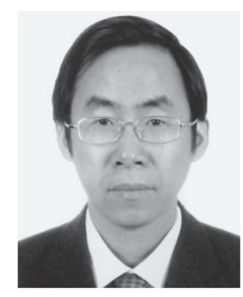

Hui Xie received the Ph.D. degree in propulsion machines and engineering from Tianjin University, Tianjin, China, in 1998. He is currently a Professor in, and the Vice Director of the State Key Laboratory of Engines, Tianjin University. His research topics are control technology for combustion processes in ICEs, theories and methods for control of gasoline HCCI/CAI engines, software and hardware for electronic control of engines and vehicles, calibration methods and technology for electronic control systems, and control of electric vehicles and hybrid electric vehicles. He has published about 100 scientific publications within the engine and vehicle field, and obtained nine patents.

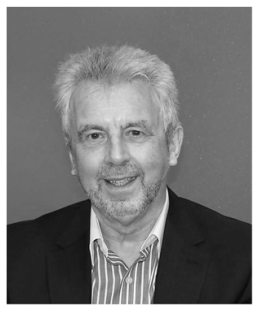

David Brown (M'10) received the Ph.D. degree in motion control from Southampton University, Southampton, U.K., in 1983. He came back to academia after spending about 20 years in industry. $\mathrm{He}$ is the author of numerous published refereed journal and conference papers in the area of artificial intelligence. His research interests include artificial intelligence and its applications to intelligent systems. Prof. Brown received an engineering excellence prize from the Royal Academy of Engineering 